\title{
Rod-like nanoparticles with striped and helical topography
}

Jani-Markus Malho, Maria Morits, Tina I. Löbling, Nonappa, Johanna Majoinen, Felix H. Schacher, Olli Ikkala, André H. Gröschel

\section{Contents:}

Materials and methods

Experimental section

Calculation of initiator density on the CNC surface

Calculation of degree of substitution

Overview and characteristics of CNC-g-PAA

Calculation for diblock copolymer chains on single CNC with different PAA chain lengths

Overview and characteristics of CNC- $g-\mathrm{PAA}_{n} / \mathrm{PMETAI}_{82}-b-\mathrm{PEO}_{114}$

Time evolution of CNC- $g$-PAA $870 / \mathrm{PMETAI}_{82}-b-\mathrm{PEO}_{114}$

Supporting references

Supporting movie 


\section{Materials and Methods}

Cellulose nanocrystals (CNCs) were obtained from cotton filter paper (Whatman 1) through sulfuric acid hydrolysis via removal of non-crystalline parts of the fibers and leaving only the rigid rod-like nanocrystals intact. The CNCs contained $0,06 \%$ of sulfate ester groups on their surface, which results from the sulfuric acid hydrolysis. The chemicals $N, N^{\prime}$ dicyclohexylcarbodiimide (DCC, $99 \%$, Fluka), 4-dimethylaminopyridine (DMAP, $99 \%$ Acros), 1,1,4,7,10,10-hexamethyltriethylenetetramine (HMTETA, $97 \%$ Aldrich), $N, N, N{ }^{\prime}, N$ ',$N$ '-Pentamethyldiethylenetriamine (PMDETA, $99 \%$ Aldrich), pyridine (99,8\% Aldrich), copper(I)bromide ( $\mathrm{CuBr}, 99,9 \%$ Acros), 2-bromoisobutyryl bromide (Br-iBBr, $98 \%$ Aldrich), ethyl $\alpha$-bromoisobutyrate (EBiB, $98 \%$ ), tert-butyl acrylate ( $t \mathrm{BA}, 98 \%$ Aldrich), 2-dimethylaminoethyl methacrylate (DMAEMA, 98\% Aldrich), trifluoroacetic acid (TFA, Riedel-de Haen) and pH 10 buffer solution (Titrinorm, VWR International) were used as received. All solvents were of analytical grade and used as received. Methyl iodide (99\%, Merck) for quaternization reactions was used without further purification. Milli-Q water purified with a Millipore filtering system was used in all cases. For dialysis, membranes of regenerated cellulose (Spectrum Laboratories, Spectra/Por MWCO = 12-14 kDa) were used.

Nuclear Magnetic Resonance. ${ }^{1} \mathrm{H}-\mathrm{NMR}$ spectra of aliquots drawn during polymerization were recorded on a Bruker AVANCE $400 \mathrm{MHz}$. Samples were diluted in deuterated chloroform $\left(\mathrm{CDCl}_{3}\right)$.

Size Exclusion Chromatography. SEC measurements of the PEO- $b$-PDMAEMA diblock copolymers were performed on a set of $30 \mathrm{~cm}$ SDV-gel columns of $5 \mathrm{~mm}$ particle size having a pore size of $10^{5}, 10^{4}, 10^{3}$, and $10^{2} \AA$ with refractive index and UV $(\lambda=254 \mathrm{~nm})$ detection. SEC was measured at an elution rate of $1 \mathrm{~mL} / \mathrm{min}$ with THF as eluent using PMMA standards. SEC measurements of free poly(tert-butylacrylate) (PtBA) homopolymer formed by sacrificial initiator were performed on a set of three Styragel columns (HR2, HR4, HR6) and a differential refractometer (Waters 410, Rochester, MN). THF was used as an eluent with a flow rate of $0.8 \mathrm{~mL} / \mathrm{min}$ and poly(methyl methacrylate) (PMMA) as standards for calibration.

Zeta-potential. Measurements were performed with disposable capillary cells (DTS1061) on a Malvern Zetasizer, using approximately $800 \mu \mathrm{L}$ of the aqueous CNC-g-PAA solutions. 
The $\zeta$-potential was calculated from electrophoretic mobility applying the Smoluchowski equation.

Cryo-transmission electron microscopy (Cryo-TEM). Cryo-TEM imaging was carried out using JEM 3200FSC field emission microscope (Jeol) operated at $300 \mathrm{kV}$ in bright field mode. Images were acquired with a CCD camera (Gatan) using an Omega-type Zero-loss energy filter, while the specimen temperature was maintained at $-187{ }^{\circ} \mathrm{C}$. Vitrified samples were prepared using FEI Vitrobot Mark IV by placing $3 \mu \mathrm{L}$ sample solution on 400 mesh lacey or holey carbon copper grids under $100 \%$ humidity, then blotted two times with filter paper for $1.5-2.5 \mathrm{~s}$ and subsequently plunged into $-170{ }^{\circ} \mathrm{C}$ ethane/propane mixture. The vitrified samples were then cryo-transferred to the microscope.

\section{Cryogenic Transmission Electron Tomography (cryo-ET) and tomographic} reconstruction. Cryo-ET tilt series were acquired with the SerialEM software package (version 3.2.2). Samples were tilted between $\pm 69^{\circ}$ angles with $2-3^{\circ}$ increment steps. For image alignment purposes, the TEM grids were coated with fiducial markers by dipping in a solution of gold nanoparticles in methanol before sample deposition $(d=3-10 \mathrm{~nm}$, stabilized by 11-mercapto-1-undecanol ligand). ${ }^{1}$ Prealignment of tilt image series was done with $\mathrm{IMOD}^{2}$ and the fine alignment and cropping with custom-made Silicon Graphics JPEGANIM SGI software package. ${ }^{3}$ The images were binned twice to reduce noise and computation time. Maximum entropy method (MEM) reconstruction scheme was carried out with custom-made script with regularization parameter value of $\lambda=1.0 \mathrm{e}^{-3}$. Volumetric graphics and analyses were performed with the UCSF Chimera package. ${ }^{4}$ The isosurface models of the particles were prepared by using Trainable Weka Segmentation ${ }^{5}$ plug-in for the FIJI open-source software package ${ }^{6}$ and then consequently low-pass filtered with Chimera's Gaussian filter with 5.0 voxel radius.

Atomic Force Microscopy (AFM). AFM measurements were performed on a Veeco Dimension 500 SPM. Samples were prepared by dip-coating of a dilute CNC-g-PAA buffer pH 10 solution $(0.2 \mathrm{~g} / \mathrm{L})$ onto a silicon wafer. After drying, the wafer was dipped into Milli-Q water to remove salt contamination resulting from the buffer solution.

Elemental Analysis (EA). EA was performed by microanalysis service at Mikroanalytisches Labor Pascher, Germany. 
Fourier Transform-infrared spectroscopy. FT-IR spectra were recorded using a Nicolet 380 instrument equipped with an ATR cell.

\section{Experimental Section}

CNC modification. The CNCs were modified with the ATRP initiator $\alpha$-bromoisobutyryl bromide in a two-step process according to the reported procedure by Majoinen et al. ${ }^{7}$ First, a $\mathrm{CNC}$ aerogel was produced by freeze drying $100 \mathrm{~mL}$ of an aqueous dispersion of sulfate CNC nanocrystals $(1.37 \mathrm{w} / \mathrm{v} \%)$. The aerogel was subsequently esterified with 2bromoisobutyryl bromide (Br-iBBr) via chemical vapor deposition (CVD). For the second esterification step, the pre-modified CNCs were dispersed in anhydrous DMF $(c(\mathrm{CNC})=10$ $\mathrm{g} / \mathrm{L})$ with $2 \mathrm{~mL}$ of pyridine and a catalytic amount of DMAP. The reaction mixture was cooled to $0^{\circ} \mathrm{C}$ in an ice bath, followed by dropwise addition of $\mathrm{Br}-\mathrm{BBBr}(2 \mathrm{~mL})$. The reaction mixture was then stirred at room temperature for $48 \mathrm{~h}$. The esterified CNCs were repeatedly washed with $\mathrm{DMF}, \mathrm{MeOH}$ and acetone in 3 centrifugation cycles for each solvent at 8000 rpm for $15 \mathrm{~min}$. The residue was finally purified by Soxhlet extraction using 1,2dichloromethane, redispersed in 1,4-dioxane and freeze-dried under vacuum to result a white powder. Esterification was followed by FT-IR (carbonyl stretching at $1743 \mathrm{~cm}^{-1}$ ) and elemental analysis showed the final bromine content of $6.0 \%$ for final initiator modified CNCs.
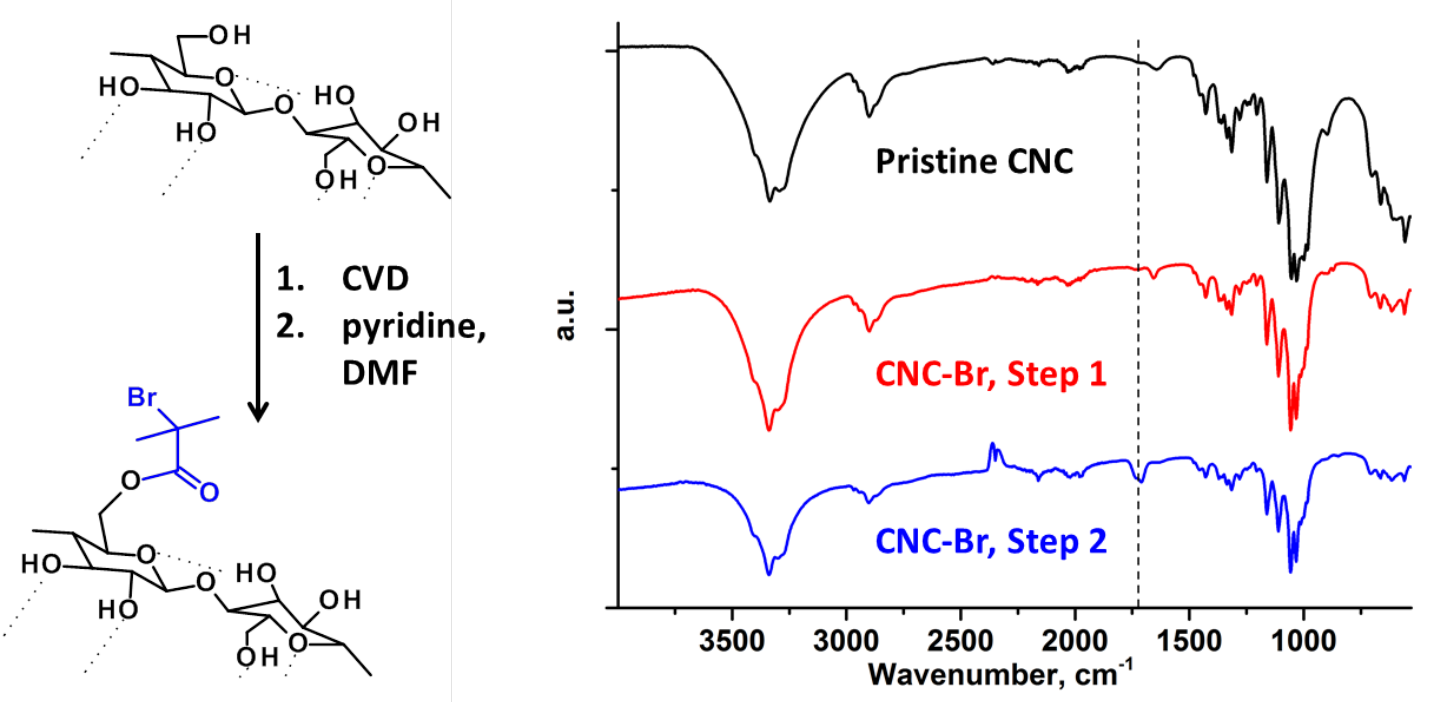

Figure S1: Schematic of modification of CNC surface and IR spectra of CNCs at different steps of modification with the ATRP initiator. 
ATRP of CNC-g-PtBA. The polymerization of $\mathrm{P} t \mathrm{BA}$ was performed using a modified recipe of the previous report. ${ }^{7}$ The reaction was carried out in DMF with $\mathrm{Cu}(\mathrm{I}) \mathrm{Br} / \mathrm{PMDETA}$ as the catalyst system. In a typical reaction, $40 \mathrm{mg}(0.032 \mathrm{mmol})$ of CNC-Br were dispersed in $5 \mathrm{ml}$ DMF. To this mixture $7.5 \mathrm{mg}(0.052 \mathrm{mmol})$ of $\mathrm{Cu}(\mathrm{I}) \mathrm{Br}, 3.9 \mathrm{mg}(0.020 \mathrm{mmol})$ of ethyl $\alpha-$ bromoisobutyrate (as sacrificial initiator) and $10 \mathrm{~g}(78 \mathrm{mmol})$ of $t$ BA were first mixed with the $\mathrm{CNC}-\mathrm{Br}$ dispersion in a $25 \mathrm{ml}$ round-bottom flask equipped with a stir bar. A solution of $9 \mathrm{mg}(0.052 \mathrm{mmol}) \mathrm{N}, N, N$ ', $N$ ', $N$ ''-pentamethyldiethylenetriamine in $1 \mathrm{ml}$ DMF was prepared in a separate $2 \mathrm{ml}$ flask. The molar ratios used for the monomer, sacrificial initiator and $\mathrm{CNC}-\mathrm{Br}$ macroinitiator and for the $\mathrm{Cu}(\mathrm{I}) \mathrm{Br} / \mathrm{PMDETA}$ catalyst system were $\left[\mathrm{M}_{0}\right]:[\mathrm{I}+\mathrm{MI}]:[\mathrm{Cu}]:[\mathrm{L}]=1500: 1: 1: 1$. Both flasks were closed with rubber septa and flushed with nitrogen for $20 \mathrm{~min}$ to remove traces of oxygen. The reaction was started after placing the flask into an oil bath heated to $70{ }^{\circ} \mathrm{C}$ and addition of the degassed solution of ligand to the mixture. The polymerization was performed at $70{ }^{\circ} \mathrm{C}$ under stirring. The polymerizations were carried out with increasing time periods to obtain different $\mathrm{P} t \mathrm{BA}$ brush lengths with increasing molecular weights (see Table S1). The monomer conversion, $x_{P}$, was determined and followed during the reaction by ${ }^{1} \mathrm{H}$ NMR and SEC of free P $t$ BA (produced by sacrificial initiator) was measured to evaluate the final brush length (Table S1). Figure S2 shows the first order kinetic plot of monomer conversion versus reaction time and confirms the controlled character of the performed ATRP reaction.

Table S1. SI-ATRP of $t$ BA from CNC-Br.

\begin{tabular}{|c|c|c|c|c|c|}
\hline Sample & $\boldsymbol{x}_{\mathbf{P}}[\mathbf{\%}]$ & $\mathbf{D P}[\mathbf{P t} \boldsymbol{t} \mathbf{B A}]$ & $\mathbf{M}_{\mathbf{n}}[\mathbf{k g} / \mathbf{m o l}]$ & $\mathbf{M}_{\mathbf{w}}[\mathbf{k g} / \mathbf{m o l}]$ & $\mathbf{P D I}$ \\
\hline $\mathrm{CNC} g-\mathrm{PAA}_{370}$ & 24 & 373 & 53.0 & 66.3 & 1.25 \\
\hline $\mathrm{CNC}_{-}-\mathrm{PAA}_{780}$ & 49.8 & 781 & 111.0 & 151.1 & 1.36 \\
\hline $\mathrm{CNC}-\mathrm{g}-\mathrm{PAA}_{870}$ & 55 & 868 & 123.5 & 153.6 & 1.24 \\
\hline
\end{tabular}




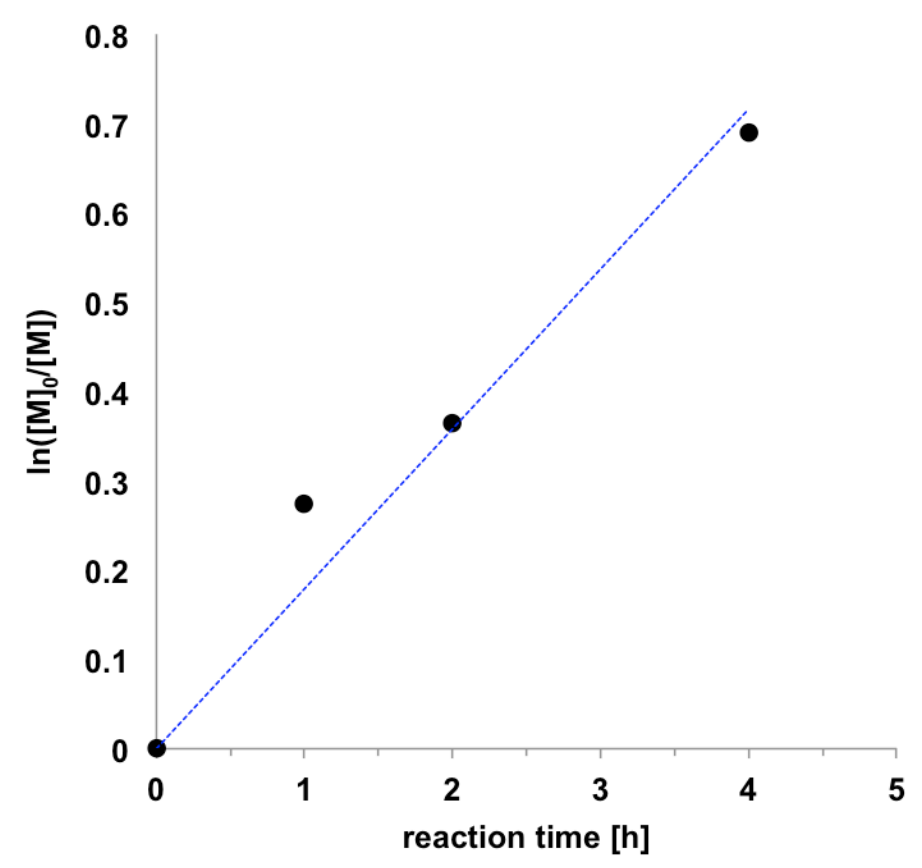

Figure S2: Semilogarithmic plot of monomer conversion versus reaction time determined by ${ }^{1} \mathrm{H}$ NMR. The linear first order kinetic plot confirms the controlled character of the surface initiated ATRP.

Purification of CNC-g-PtBA from free PtBA. Free P $t$ BA produced by sacrificial initiator was separated from the grafted $\mathrm{CNC}-g-\mathrm{P} t \mathrm{BA}$ via centrifugation at $5000 \mathrm{rpm}$ for $5 \mathrm{~min}$ using $\mathrm{THF}$ as solvent. The procedure was repeated twice. CNC- $g-\mathrm{P} t \mathrm{BA}$ material was cleaned by dispersing in isopropanol followed by centrifugation at $20,000 \mathrm{rpm}$ for $20 \mathrm{~min}$. The centrifugation procedure was repeated three times. The clean $\mathrm{CNC}-g$-P $t \mathrm{BA}$ samples were dried in vacuum oven at $20^{\circ} \mathrm{C}$ over night to give a white solid. The supernatants containing $\mathrm{P} t \mathrm{BA}$ homopolymer were dried by evaporating the solvent followed by drying in vacuum oven at $20^{\circ} \mathrm{C}$ over night. The dry P $t$ BA samples were dispersed in THF at a concentration 10 $\mathrm{mg} / \mathrm{ml}$ and passed through silica 60 column in order to purify samples from copper. The purified samples were characterized via SEC in THF using PMMA standards to evaluate the molecular weight and PDI.

Hydrolysis to CNC-g-PAA . $10 \mathrm{mg}$ of CNC-g-P $t$ BA was dispersed in $3 \mathrm{~mL}$ of DCM followed by drop-wise adding of 10 eq. excess trifluoroacetic acid (TFA) compared to the $t$ BA-groups. The reaction mixture was stirred for $24 \mathrm{~h}$ at room temperature. TFA and DCM were removed by drying the sample at $50{ }^{\circ} \mathrm{C}$ under vacuum. Figure S2 shows the FT-IR spectra before and after deprotection of the PtBA brush to PAA for the sample CNC- $g$ $\mathrm{P}_{t} \mathrm{BA}_{870}$. The peak of the $\mathrm{CH}$ deformation of tert-butyl-group at $1368 \mathrm{~cm}^{-1}$ vanishes almost 
entirely for the deptrotected sample. A broad peak from the carboxlic acid O-H stretching is visible at $2500-3000 \mathrm{~cm}^{-1}$ for $\mathrm{CNC}_{-}-\mathrm{PAA}_{870}$ (red curve). The slight shift to lower wave numbers (from $1725 \mathrm{~cm}^{-1}$ to $1700 \mathrm{~cm}-1$ ) of the carbonyl peak further confirms the successful hydrolysis of PtBA to PAA. After removal of TFA and DCM, CNC-g-PAA $n$ was transferred to a buffer solution ( $\mathrm{pH} \mathrm{10)} \mathrm{with} \mathrm{an} \mathrm{approx.} \mathrm{salt} \mathrm{concentration} \mathrm{of} 50 \mathrm{mM}$. Salt suppresses the strong electrosteric brush-brush repulsion of negatively charged PAA brushes $(\zeta=-30 \mathrm{mV})$ and significantly reduces the viscosity of $\mathrm{CNC}-\mathrm{g}-\mathrm{PAA}_{\mathrm{n}}$ dispersions (relevant for further characterization in cryo-TEM).

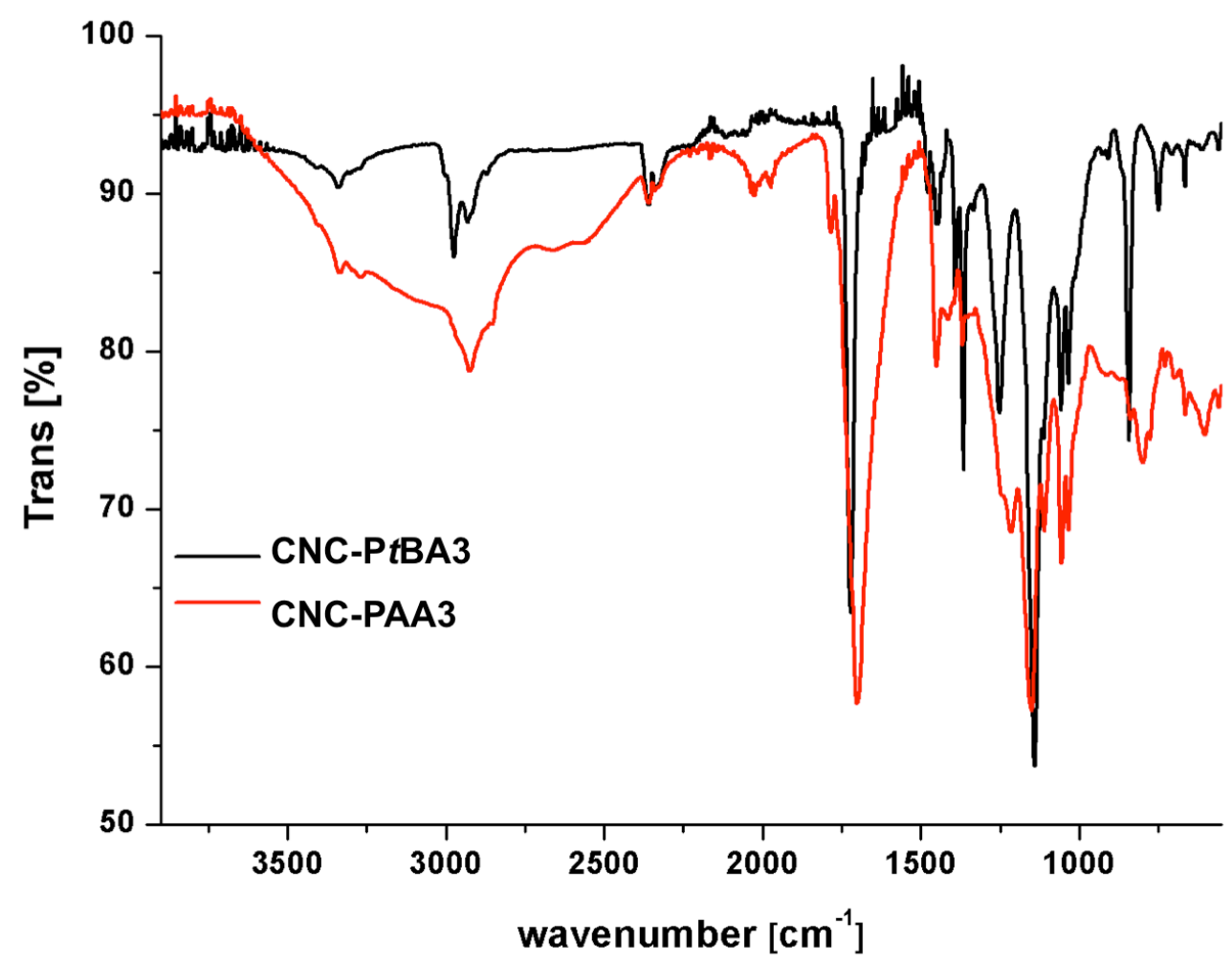

Figure S3: FT-IR spectra before (black) and after (red) deprotection of the PtBA brush to PAA exemplified for the sample CNC- $g$-PtBA 870 .

Synthesis of PEO- $b$-PDMAEMA. The ATRP of the PEO- $b$-PDMAEMA diblock copolymer was performed according to a previous publication. ${ }^{8}$ In brief, the $\mathrm{PEO}_{114}$-Br macroinitiator was synthesized via Steglich esterification reacting monomethoxypoly(ethylene glycol) $\left(M_{\mathrm{n}}=5000 \mathrm{~g} / \mathrm{mol}, \mathrm{PDI}=1.02\right)$ with 2-bromoisobutyric acid in DCM in the presence of DCC and a catalytic amount of DMAP. The $\mathrm{PEO}_{114} \mathrm{Br}$ macroinitiator was obtained by recrystallization from ethanol as a white powder. For the synthesis of PEO-b-PDMAEMA, $\mathrm{PEO}_{114} \mathrm{-Br} \quad(0.3 \mathrm{~g}, \quad 0.15 \mathrm{mmol}), \quad \mathrm{CuCl} \quad(0.015 \mathrm{~g}, 0.015 \mathrm{mmol}), \quad$ DMAEMA $(1.767 \mathrm{~g}$, $11.24 \mathrm{mmol}$ ) and $4 \mathrm{~mL}$ acetone were added to a $10 \mathrm{~mL}$ screw cap glass, equipped with a 
magnetic stir bar and degassed with nitrogen for $20 \mathrm{~min}$ to remove traces of oxygen from the solution. In a separate vial, $0.081 \mathrm{~g}(0.3 \mathrm{mmol})$ of the ligand HMTETA was dissolved in 2 $\mathrm{mL}$ acetone and degassed with nitrogen before transferring to the $10 \mathrm{~mL}$ screw cap glass with a gas-tight, nitrogen-flushed syringe. The reaction mixture transferred to an oil bath preheated to $50{ }^{\circ} \mathrm{C}$ and stirred for a specific time before terminating the reaction by exposure to air and rapid cooling in liquid nitrogen. After dilution with THF, the mixture was passed through a silica column to remove the catalyst and precipitated in cold n-hexane $\left(-30^{\circ} \mathrm{C}\right)$ followed by freeze-drying from dioxane. A PDI of 1.17 was determined by SEC measurements in THF using PEO as standards. The composition of $\mathrm{PEO}_{114}-b$-PDMAEMA 82 was calculated from ${ }^{1} \mathrm{H}-\mathrm{NMR}$ measurements.

Quaternization of PEO-b-PDMAEMA to PEO-b-PMETAI. 50 mg of PEO- $b$-PDMAEMA were dissolved in $5 \mathrm{~mL}$ deionized water and 10 eq. of methyl iodide (compared to DMAEMA units) were added. The mixture was allowed to stir for two days at room temperature. Afterward, the solution was dialyzed two days against dioxane/water 80:20 v/v followed by dialysis against dioxane/water 50:50 v/v for two days. The quaternized $\mathrm{PEO}_{114^{-}}$ $b$-PMETAI 82 was obtained as a white powder after freeze-drying.

IPEC formation. To $0.5 \mathrm{~mL}$ of a $0.2 \mathrm{~g} / \mathrm{L}$ CNC-g-PAA $A_{n}$ buffer solution (pH 10), a predetermined amount of a $2 \mathrm{~g} / \mathrm{L}$ PEO- $b$-PMETAI buffer solution ( $\mathrm{pH}$ 10) was added under vigorous stirring to reach stoichiometric conditions, i.e., $n(\mathrm{AA}): n(\mathrm{Dq})=1: 1$. The mixture was allowed to equilibrate under stirring for at least $5 \mathrm{~d}$ prior to analysis. 


\section{Calculation of initiator density on the CNC surface}

The bromine content of $f_{\mathrm{Br}}=6.5 \mathrm{wt} .-\%$ for the final initiator modified CNCs was determined by elemental analysis. The molar concentration of bromine $n(\mathrm{Br})$ is equal to the molar concentration of initiator $n$ (Ini) and given by:

$$
n(\mathrm{Ini})=\frac{f_{B r}}{M(B r) * 100 \%}=\frac{6.5 \%}{79.9 \frac{\mathrm{g}}{\mathrm{mol}} * 100 \%}=0.81 * 10^{-3} \frac{\mathrm{mol}}{\mathrm{g}}
$$

where $M(\mathrm{Br})=79.9 \mathrm{~g} / \mathrm{mol}$ is the molecular weight of bromine. Considering the molecular weight per initiator, $M(\mathrm{Ini})=150 \mathrm{~g} / \mathrm{mol}$, the initiator weight fraction equals

$$
f_{\text {Ini }}=n(\operatorname{Ini}) * M(\operatorname{Ini})=0.00081 * 150 \frac{\mathrm{g}}{\mathrm{mol}}=0.12
$$

The molar amount of anhydroglucose units per gram with molecular weight $M(A n G l u)=162 \mathrm{~g} / \mathrm{mol}$ is given by:

$$
n(\text { AnGlu })=\frac{1-f_{\text {Ini }}}{M(\text { AnGlu })}=\frac{1-0.12}{162}=0.54 * 10^{-2} \frac{\mathrm{mol}}{\mathrm{g}}
$$

The molar ratio of initiator to anhydroglucose (AnGlu) units in the material then results:

$$
\frac{n(\text { Ini })}{n(\text { AnGlu })}=\frac{0.81 * 10^{-3} \frac{\mathrm{mol}}{\mathrm{g}}}{0.54 * 10^{-2} \frac{\mathrm{mol}}{\mathrm{g}}}=0.15
$$

In order to estimate the density of the initiator on the CNC surface we will simplify a CNC as a cylindrical rod with radius of $r \approx 3.7 \mathrm{~nm}$ and segment length of $h .^{7}$ The initiator density on the surface of such segment of CNC is given by:

$$
\begin{gathered}
N_{\text {surf_Ini }}=\frac{\rho(\text { AnGlu }) * V_{C y l} * n(\text { Ini }) * N_{A}}{M(\text { AnGlu }) * S_{C y l} * n(\text { AnGlu })}=\frac{\rho(\text { AnGlu }) * r * N_{A}}{M(\text { AnGlu }) * 2} * \frac{n(\text { Ini })}{n(\text { AnGlu })} \\
=\frac{1.58 * 10^{-21} \frac{g}{n^{3}} * 3.7 \mathrm{~nm} * 6.022 * 10^{23} \frac{1}{\mathrm{~mol}}}{162 \frac{\mathrm{g}}{\mathrm{mol}} * 2} * 0.15=1.6 \frac{1}{\mathrm{~nm}^{2}}
\end{gathered}
$$

The density of crystalline cellulose is $\rho(\mathrm{AnGlu})=1.58 \mathrm{~g} / \mathrm{cm}^{3}=1.58 * 10^{-21} \mathrm{~g} / \mathrm{nm}^{3}, V_{\text {cyl }}=r^{2} \pi h$ is the volume of cylinder segment, $N_{A}=6.022 * 10^{23} \mathrm{~mol}^{-1}$ is the Avogadro constant and $S_{\text {cyl }}=r 2 \pi h$ is surface area of the cylinder segment. 


\section{Calculation of degree of substitution}

Using the approximation that we have $I_{\beta}$ cellulose with lattice plane spacing of $x=0.53 \mathrm{~nm}$ and $y=0.61 \mathrm{~nm}^{9,10}$ we can assume the number of cellulose chains on the surface, $N_{\text {surf }}$, for a given $\mathrm{CNC}$ cylindrical segment $(r=3.7 \mathrm{~nm})$ :

$$
N_{\text {surf }}=\frac{4 r}{x}+\frac{4 r}{y}=2 * 3.7 n m *\left(\frac{1}{0.53 \mathrm{~nm}}+\frac{1}{0.61 \mathrm{~nm}}\right) \approx 52
$$

The total number of cellulose units per cross-section, $N_{\text {all }}$ :

$$
N_{\text {all }}=\frac{\pi r^{2}}{x * y}=\frac{3.14 *(3.7 \mathrm{~nm})^{2}}{0.53 \mathrm{~nm} * 0.61 \mathrm{~nm}} \approx 133
$$

Surface chains number ratio to all chains number is

$$
\frac{N_{\text {surf }}}{N_{\text {all }}}=\frac{52}{133} \approx 0.39
$$

The degree of substitution $D S_{C N C}$ calculates to:

$$
D C_{C N C}=\frac{N_{\text {all }}}{N_{\text {surf }}} * \frac{n(\text { Ini })}{n(\text { AnGlu })}=\frac{1}{0.39} * 0.15 \approx 0.4
$$


Overview and characteristics of CNC-g-PAA
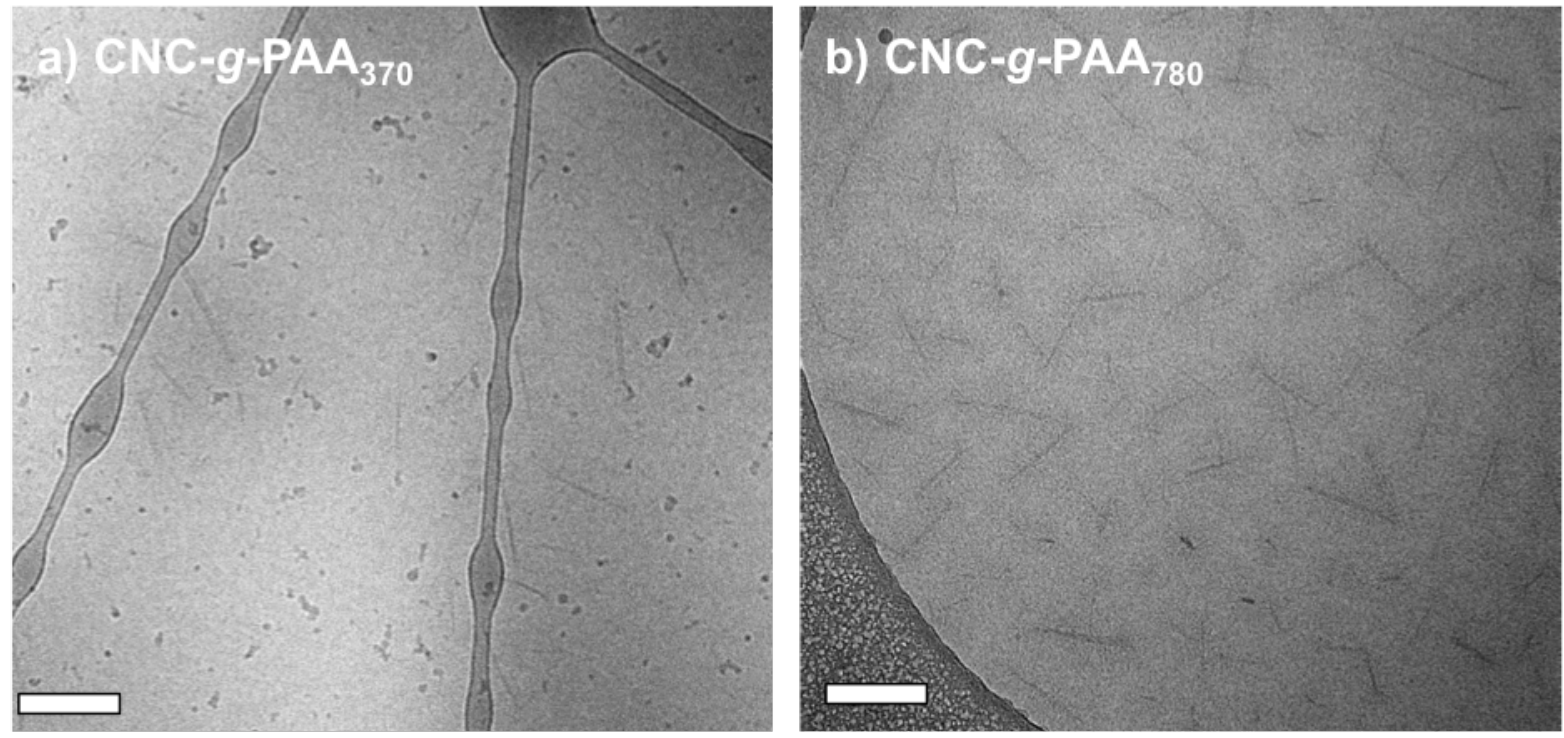

c) $\mathrm{CNC}-g-\mathrm{PA} \mathrm{A}_{870}$

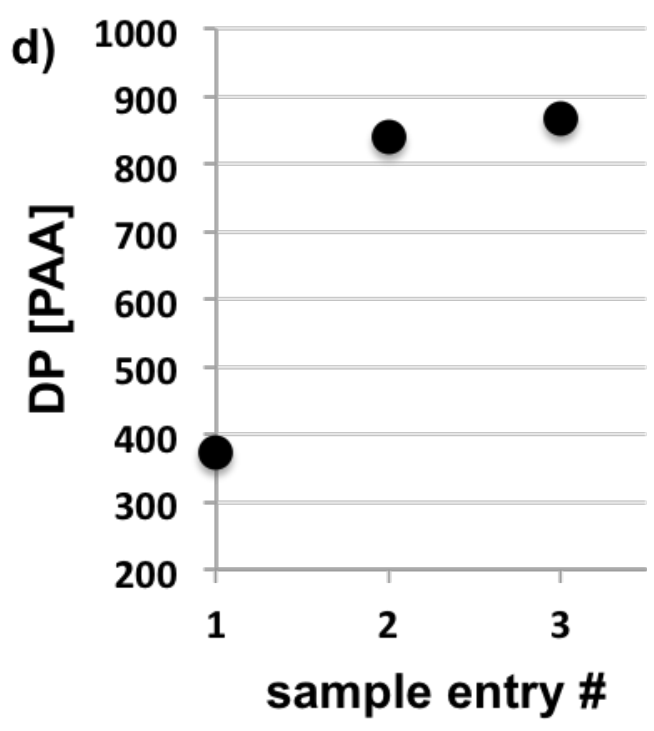

Figure S4: a-c) Cryo-TEM overview images of $\mathrm{CNC}^{-} \mathrm{g}-\mathrm{PAA}_{\mathrm{n}}$ showing $\mathrm{CNCs}$ with increasing PAA brush lengths. Scale bars are $200 \mathrm{~nm}$. d) The increase of PAA block length for the samples a-c.

Table S2. Characteristics for CNCs and CNC-g-PAA ${ }_{n}$ based on cryo-TEM images.

\begin{tabular}{|c|c|c|c|}
\hline Sample & measured value & $\begin{array}{c}\text { mean value } \\
{[\mathrm{nm}]}\end{array}$ & $\begin{array}{c}\text { standard deviation } \\
{[\mathrm{nm}]}\end{array}$ \\
\hline $\mathrm{CNC}$ & length & 124.7 & 44.5 \\
\hline CNC-g-PAA 370 & brush radius & 59.4 & 12.9 \\
\hline CNC-g-PAA 780 & brush radius & 62.7 & 7.5 \\
\hline CNC-g-PAA 870 & brush radius & 70.6 & 10.5 \\
\hline
\end{tabular}




\section{Calculation for diblock copolymer chains on single $\mathrm{CNC}$ with different PAA chain lengths}

Number of PAA chains on the single CNC surface $N(P A A)_{\text {per } C N C}$, is assumed to be equal to the number of initiator units on the surface and given by:

$$
\begin{aligned}
N(P A A)_{\text {per } C N C} & =N_{\text {Surf } \_ \text {Ini }} * S_{\text {Cyl }}=N_{\text {Surf IIni }} *\left(L * 2 \pi * r+2 * \pi * r^{2}\right) \\
& =1,6 \mathrm{~nm}^{-2} *\left(125 \mathrm{~nm} * 6,28 * 3,7 \mathrm{~nm}+2 * 3,14 * 3,7^{2} \mathrm{~nm}^{2}\right) \approx 4790
\end{aligned}
$$

Number of diblock chains needed for neutralization of one PAA brushes is given by ratio of $D P s:$

$$
\frac{D P_{P A A}}{D P_{P M E T A I}}
$$

Total number of diblock chains on the $\mathrm{CNC}$ surface, $N(\text { diblock })_{\text {per } C N C}$ :

$$
N(\text { diblock })_{\text {per } C N C}=\frac{D P_{P A A}}{D P_{P M E T A I}} * N(P A A)_{\operatorname{per~} C N C}
$$

Amounts of diblock copolymers needed for neutralization of PAA brushes with different $D P$ and number of diblock copolymers per CNC are shown in the table S3.

Table S3. Amount of diblock copolymer chains per CNC.

\begin{tabular}{|c|c|c|c|}
\hline Sample & DP(PAA) & PMETAI/PAA & diblock chains/CNC \\
\hline${\text { CNC- } g-P_{A A}}_{370} / \mathrm{PMETAI}_{82}-b-\mathrm{PEO}_{114}$ & 373 & 4.5 & 21770 \\
\hline $\mathrm{CNC}-\mathrm{g}-\mathrm{PAA}_{780} / \mathrm{PMETAI}_{82}-b-\mathrm{PEO}_{114}$ & 781 & 9.5 & 45600 \\
\hline $\mathrm{CNC}-\mathrm{PAA}_{870} / \mathrm{PMETAI}_{82}-b-\mathrm{PEO}_{114}$ & 868 & 10.6 & 50700 \\
\hline
\end{tabular}




\section{Overview and characteristics of CNC-g-PAA $/$ PMETAI $_{82}-b-$ PEO $_{114}$}
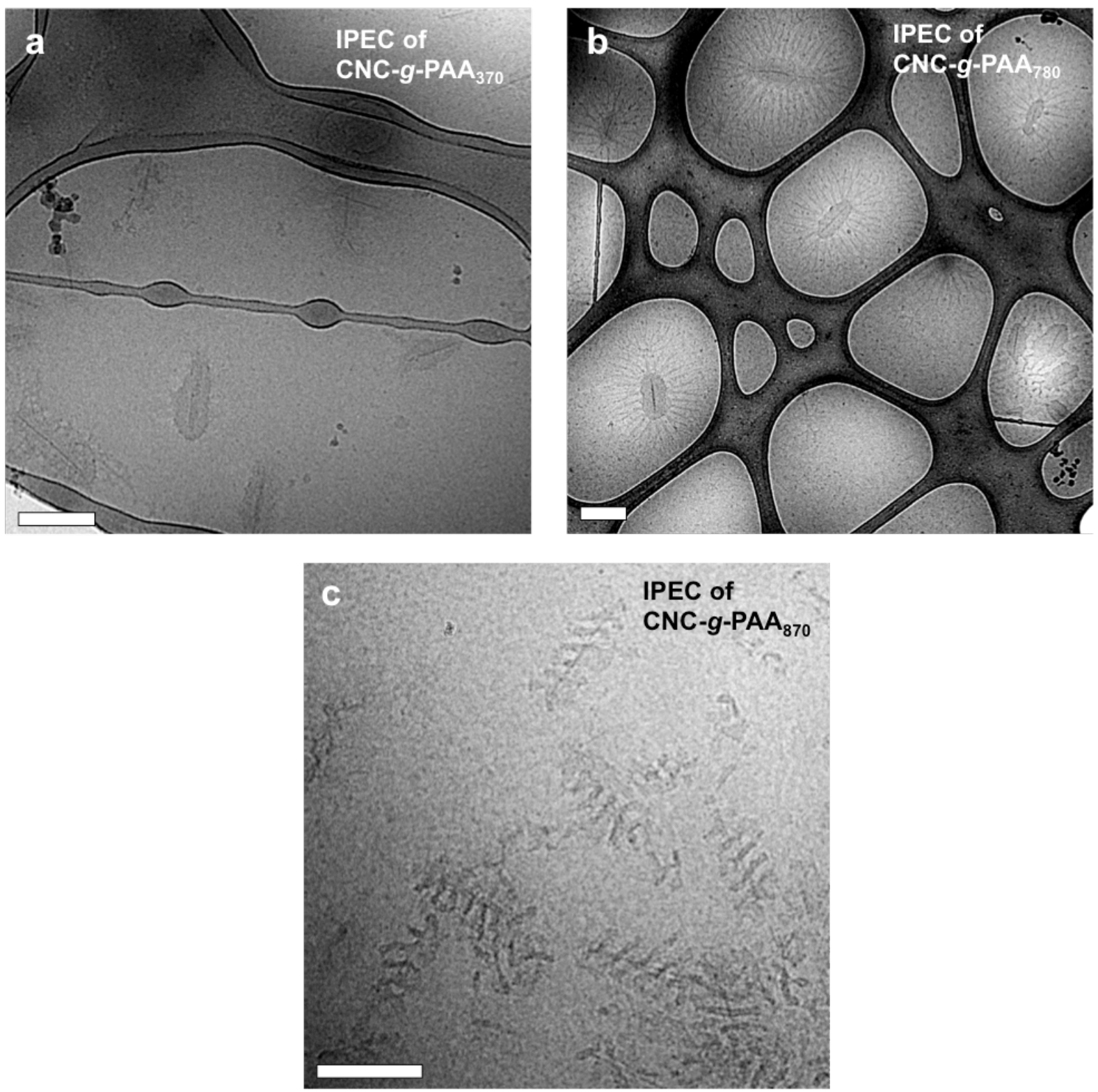

Figure S5: Cryo-TEM overview images of CNC-g-PAA $/ \mathrm{PMETAI}_{82}-b-\mathrm{PEO}_{114}$. The formed IPEC on CNCs evolves from homogeneous IPEC shell a) towards an IPEC shell with cylindrical tentacles b) and finally to helical IPEC screw c) with increasing PAA brush lengths. Scale bars are $200 \mathrm{~nm}$. 
Table S4. Characteristics of CNC-g-PAA $/{ }^{2}$ PMETAI $_{82}-b-P_{114}$.

\begin{tabular}{|c|c|c|c|}
\hline Sample & measured value & $\begin{array}{c}\text { mean } \\
{[\mathrm{nm}]}\end{array}$ & $\begin{array}{c}\text { standard } \\
\text { deviation } \\
{[\mathrm{nm}]}\end{array}$ \\
\hline CNC- $g$-PAA ${ }_{370} / \mathrm{PMETAI}_{82}-b-\mathrm{PEO}_{114}$ & IPEC shell thickness & 43.6 & 6.9 \\
\hline \multirow{2}{*}{ CNC- $g$-PAA $780 / \mathrm{PMETAI}_{82}-b-\mathrm{PEO}_{114}$} & IPEC shell thickness & 48.1 & 7.8 \\
\hline & IPEC tentacle thickness & 9.6 & 2.2 \\
\hline \multirow{2}{*}{ CNC- $g$-PAA ${ }_{870} / \mathrm{PMETAI}_{82}-b-\mathrm{PEO}_{114}$} & IPEC lamella length & 48.2 & 9.1 \\
\hline & IPEC lamella thickness & 14.3 & 2.2 \\
\hline
\end{tabular}




\section{Time evolution of CNC-g-PAA $870 /$ PMETAI $_{82}-b-P_{114}$}

By cryo-TEM analysis of the same sample at different stages of IPEC formation, we identify two stages of helical IPEC formation. First, due to kinetical constrains, thin IPEC shell forms around grafted $\mathrm{CNC}$ and shields non-neutralized PAA units from the rest of the PEO- $b$ PMETAI (Figure S6a). After this initial aggregation, the polymer chains relax and diblock copolymer chains diffuse deeper into PAA brushes shell. As a result, when PEO- $b$-PMETAI reach $\mathrm{CNC}$ core and most of PAA brushes are neutralized these intermediate amoeba-like morphologies equilibrate into the more pronounced, rigid screw-like IPEC morphologies after ageing for at least one week (Figure S6b).

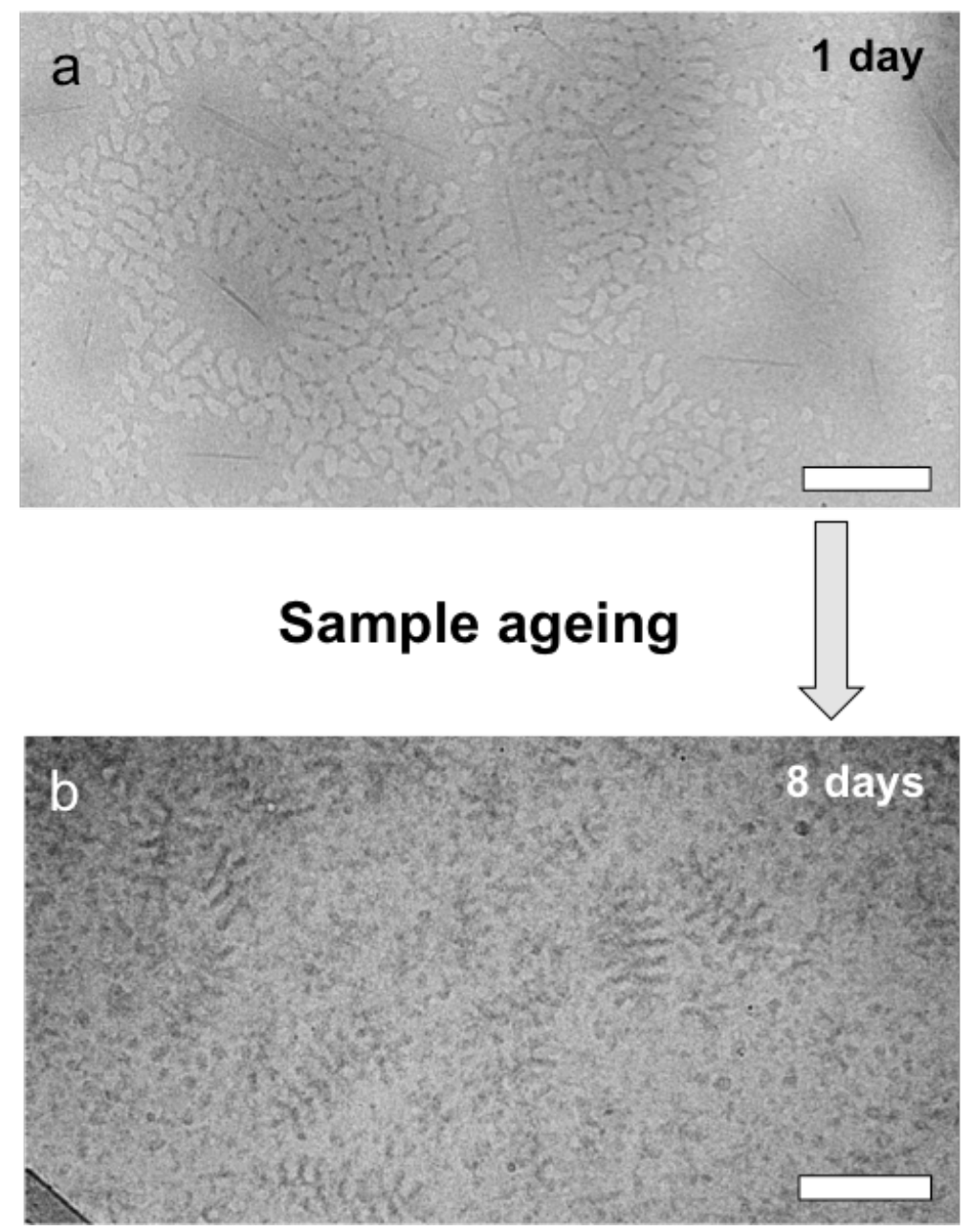

Figure S6: Time evolution of CNC-g-PAA $870 / \mathrm{PMETAI}_{82}-b-\mathrm{PEO}_{114}$. a) First an irregular IPEC shell with tentacles form after one day of mixing. b) After 8 days the structure has relaxed into a helical IPEC screw. The scale bar is $200 \mathrm{~nm}$. 


\section{Supporting References}

(1) Raula, J.; Shan, J.; Nuopponen, M.; Niskanen, A.; Jiang, H.; Kauppinen, E. I.; Tenhu, H. Langmuir 2003, 19, 3499.

(2) Kremer, J. R.; Mastronarde, D. N.; McIntosh, J. R. J. Struct. Biol. 1996, 116, 71.

(3) Engelhardt, P. Electron Tomography of Chromosome Structure; 2000.

(4) Pettersen, E. F.; Goddard, T. D.; Huang, C. C.; Couch, G. S.; Greenblatt, D. M.; Meng, E. C.; Ferrin, T. E. J. Comput. Chem. 2004, 25, 1605.

(5) Hall, M.; Frank, E.; Holmes, G.; Pfahringer, B.; Reutemann, P.; Witten, I. H. ACM SIGKDD Explor. 2009, 11, 10.

(6) Johannes Schindelin; Arganda-Carreras, I.; Frise, E.; Kaynig, V.; Longair, M.; Pietzsch, T.; Preibisch, S.; Rueden, C.; Saalfeld, S.; Schmid, B.; Tinevez, J.-Y.; White, D. J.; Hartenstein, V.; Eliceiri, K.; Tomancak, P.; Cardona, A. Nat. Methods 2012, 9, 676.

(7) Majoinen, J.; Walther, A.; McKee, J. R.; Kontturi, E.; Aseyev, V.; Malho, J. M.; Ruokolainen, J.; Ikkala, O. Biomacromolecules 2011, 12, 2997.

(8) Löbling, T. I.; Haataja, J. S.; Synatschke, C. V.; Schacher, F. H.; Müller, M.; Hanisch, A.; Gröschel, A. H.; Müller, A. H. E. ACS Nano 2014, 8, 11330.

(9) Moon, R. J.; Martini, A.; Nairn, J.; Simonsen, J.; Youngblood, J. Chem. Soc. Rev. 2011, 40, 3941.

(10) Rosilo, H.; Kontturi, E.; Seitsonen, J.; Kolehmainen, E.; Ikkala, O. Biomacromolecules 2013, 14, 1547.

\section{Supporting movies}

IPEC-single-screw-new.mpg: The video was recorded on chimera 3D reconstruction of CNC$g$-IPEC ${ }_{11}$ showing a helical IPEC topography winding around the $\mathrm{CNC}$ backbone. The video shows the particle rotating $180^{\circ}$ around the z-axis.

IPEC-screw-double.mpg: The video was recorded on chimera 3D reconstruction of CNC-gIPEC $_{11}$ showing a double-helical IPEC topography winding around the $\mathrm{CNC}$ backbone. The video shows the particle rotating $180^{\circ}$ around the $\mathrm{z}$-axis. 